\title{
Network analysis of core and associated symptoms in preschool children with autism spectrum disorder
}

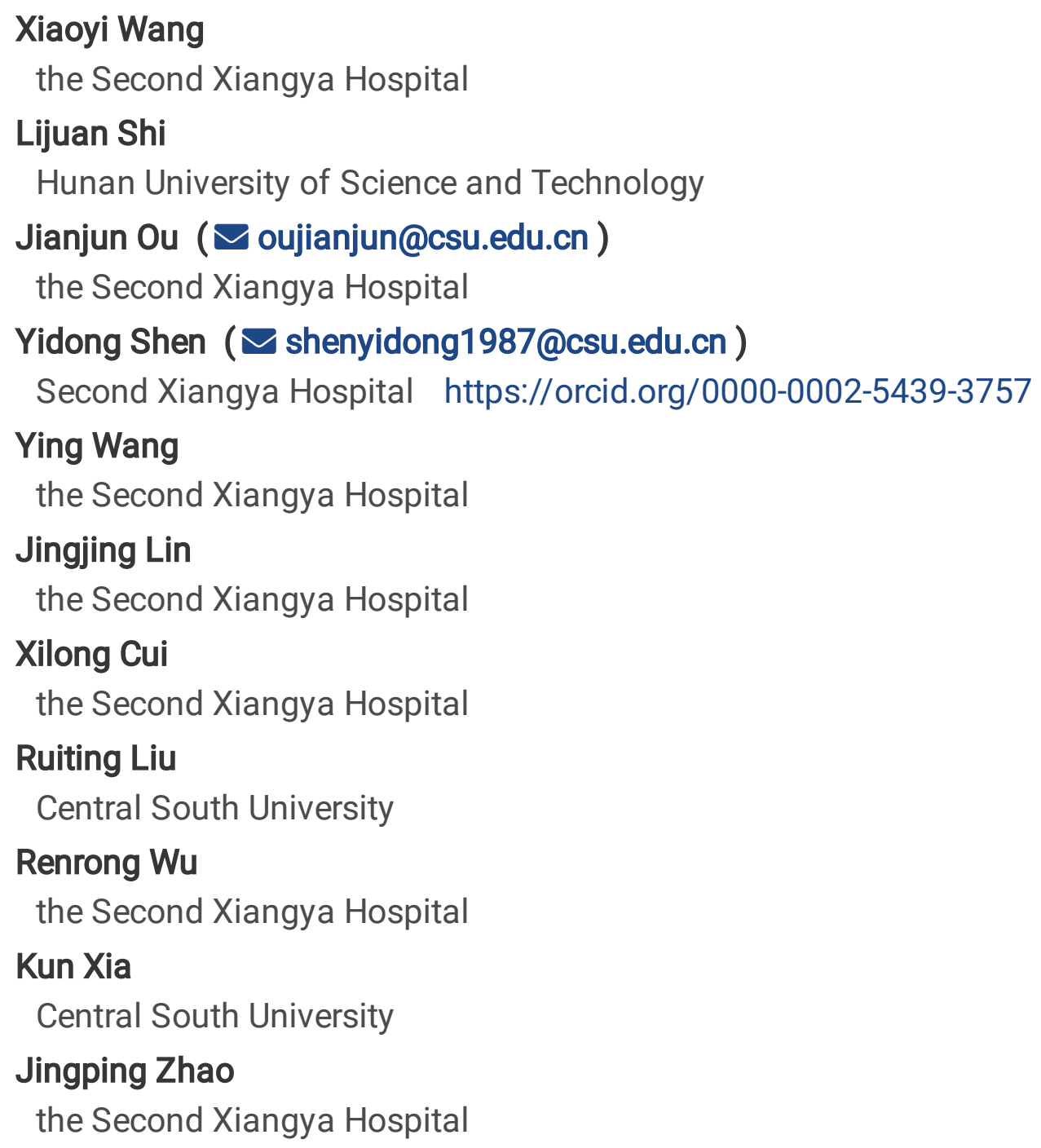

\section{Research}

Keywords: Autism Spectrum Disorder, Network Analysis, Core Symptoms, Associated Symptoms

Posted Date: August 1st, 2020

DOI: https://doi.org/10.21203/rs.3.rs-48456/v1 
License: (c) (i) This work is licensed under a Creative Commons Attribution 4.0 International License. Read Full License 


\section{Abstract}

Background: Complex relationships may exist in the symptoms of autism spectrum disorder (ASD) and may interfere with each other. Previous studies investigate the relationships between core and associated symptoms. However, there is still a lack of holistic view, especially in the relationships between core and associated symptoms.

Methods: Data were collected from a sample of 474 children with ASD. The Social Responsiveness Scale (SRS) was used to evaluate the existence and severity of autistic core symptoms of social impairment, Children's Sleep Habits Questionnaire (CSHQ) to sleep status, and Child Behavior Checklist (CBCL/1.5-5) to behavior performance of subjects. A network analysis approach was performed to assess the partial relationships of these symptoms.

Results: The social communication problem was the most central symptom of ASD. There were broad relationships between the core and associated symptoms, such as the relationships between autistic core symptoms and characteristic symptoms of ADHD and within associated symptoms. All these correlations were positive, except for a negative relationship between anxious/depressed problems and social awareness. The associations between some of these symptoms were stronger than the other associations, and the relationship between emotional problems and aggressive behavior was relatively stronger than the other relationships. The strength centrality and network edges were highly stable.

Limitations: Two main limitations exist in current study. First, the network analyses was conducted by using cross-sectional data, so it remains unknown that whether the network structure will change with the progress of the course of the disease or the change of the severity of symptoms. Second, some nodes may actually be measuring overlapping constructs, which could artificially inflate edge weights and centrality.

Conclusions: An overall perspective of connections of symptoms of ASD using network analysis indicated that controlling these associated symptoms may improve core symptoms, such as ADHDrelated symptoms. It may help identify new ways to improve symptoms in ASD.

\section{Introduction}

Autism spectrum disorder (ASD), the most common and representative disorder of pervasive developmental disorders (PDD), originates mainly in infants and young children, with social communication disorders, limited interests, and stereotyped and repetitive behavior as the main clinical manifestations[1]. In addition to these core symptoms, however, children with ASD suffer from sleep problems, emotional problems, and broad behavioral problems, such as anxiety or depression, inattention, withdrawal, and aggression. It is well documented that sleep problems are very common in children with ASD, although its prevalence varied from study to study, even with up to over $80 \%[2]$. Emotional and behavioral problems have also been found to co-occur in children with ASD, and the coprevalence is much higher than that of typically developed children[3, 4]. It is common for the co- 
occurrence of attention-deficit hyperactivity disorder (ADHD) and ASD with a co-prevalence of $28.2 \%$ (95\% $\mathrm{Cl}$ 13.3-43.0)[5], and patients with autism often manifest some clinical features of ADHD, such as attention-deficit. Meanwhile, a considerable number of patients with ASD often exhibit aggressive behaviors (including self-aggression and other-aggression), as reported that nearly one-third of the children with ASD displayed aggression that may disturb the parents or caregivers and may lead to a high risk of hospitalization and worse prognosis at the same time, resulting in an increasing burden on family and society[6, 7].

The relationships between core and associated symptoms or comorbidities and within associated symptoms have been partly explored yet lack holistic view, especially for the relationships between core and associated symptoms. Sleep disturbances are found to exacerbate autistic symptoms[8]. It has also been reported that aggressive behaviors are associated with sleep problems, attention problems, impulsivity, or hyperactivity. Concurrently, the severity of sleep problems is related to the severity of aggressive behavior, as the more serious the sleep disturbance, the more aggressive behavior the patients may suffer in[6, 7]. In addition, emotional and behavioral problems often interfere with each other, which can lead to the deterioration of the disorder and ultimately result in exacerbation of functional impairment and poor prognosis $[9,10]$. This means that complex relationships may exist in the symptoms of ASD and may interfere with each other, leading to increased difficulty in disorder management.

Accordingly, we designed the present study to explore the multidimensional relationships among these core symptoms and co-occurring symptoms of ASD. Network analysis[11, 12] approach was used to assess the partial relationships of these symptoms in ASD. Rooted in physics and mathematics, network analysis is an integrative approach that can be applied in psychopathological research of mental disorders by establishing a network model to study the multidimensional relationships between symptoms and disorders. For example, researchers examine the relationships between depression and anxiety symptoms using network analysis[13]. Given the difficulties in the improvement of the core autistic symptoms, our findings provide an important reference for the use of these interactions to alleviate the core symptoms by dealing with the "marginal symptoms" to improve the prognosis of children with ASD.

\section{Methods}

\section{Study Sample}

Data of children with ASD were derived from the Autism Clinical and Environmental Database (ACED) in China, which was established in 2012 by the Second Xiangya Hospital of Central South University, and had been described in detail in our previous article[14]. Children with ASD were recruited in special education schools or hospitals around China. The inclusion criteria were as follows: (1) 2-5 years old and (2) diagnosed with autism, Asperger's disorder, or pervasive developmental disorder not otherwise specified (PDD-NOS) according to the criteria of the Diagnostic and Statistical Manual of Mental Disorders, Fourth Edition, Text Revised (DSM-IV-TR), which was consistent with the diagnostic criteria of 
ASD in the DSM-V. Patients were excluded if there were any evidence of potential physical disorder, or they were diagnosed with any other psychiatric disorder or developmental disorder. Neuropsychological and psychopathological evaluations were conducted by trained child psychiatrists.

This study was approved by the Ethics Committee of the Second Xiangya Hospital. The parents or legal guardians of the subjects who participated in this study signed the consent forms.

\section{Measurements}

Social Responsiveness Scale

Social Responsiveness Scale (SRS) is a 65-item rating scale that measures the severity of autistic symptomatology observed by parents or caregivers[15]. It assesses five aspects of social impairments: social awareness, social cognition, social communication, social motivation, and restricted interests and repetitive behavior. Raw scores were used in the study because of the lack of norms in China. Higher scores indicate more severe symptoms of ASD.

Children's Sleep Habits Questionnaire

Children's Sleep Habit Questionnaire (CSHQ) is a parent-report questionnaire widely used in pediatric sleep questionnaire[16]. The scale has 33 items, and each item is rated on a 3-point scale (1= never/rarely, 0-1 night per week; 2= sometimes, 2-4 nights per week; and 3= usually, 5-7 nights per week). Raw scores were used in the study, and a higher total score indicates worse sleep problems.

Child Behavior Checklist for Age 1.5-5

Child Behavior Checklist for Age 1.5-5 (CBCL/1.5-5) has been broadly used to assess preschool children's emotional and behavioral problems for a long time[17]. It lists 99 items comprising seven domains:, including emotional problems, anxious/depressed problems, somatic complaints, withdrawal, sleep problems, attention problems, and aggressive behavior; and three summary scales: internalizing, externalizing, and total problems. The scale needs parents or caregivers to rate each question on a 3point scale ( $0=$ not true, $1=$ somewhat or sometimes true, and $2=$ very true or often true). In addition to the raw score, the T-score can be transformed based on the norm of the population. Here, the T-score was used to reflect the severity of emotional and behavioral problems. The higher the score, the worse the problems.

\section{Statistical and Network Analysis}

Demographic and clinical characteristics were analyzed using SPSS version 19 (SPSS, Inc., Chicago).

The network analysis was performed using a graphical Gaussian model (GGM) in R language. We constructed the networks by using the total score of CSHQ total and subscale scores of SRS and CBCL. Each of them is symbolled as "node"; the "edges" between the "nodes" represent the partial correlation between them. The thicker/wider the edge, the stronger the correlation. Green edges indicate positive 
correlation, while pink edges show negative correlations. Apart from the "edges", we also estimated the central estimation and stability of the network.

\section{Results}

A total of 474 participants with ASD were recruited in our study. Table 1 summarizes the clinical and demographic characteristics of all subjects. These patients were all preschool children aged 2-5 years, of which male children were predominant. The subscores of the three scales are shown in Table 1 in the form of "Mean (Standard Deviation)."

\section{Network Analysis}

\section{Characteristics of the network structure}

Figure 1 shows the network structure of subjects with ASD. Results showed a well-connected network, since there were no isolated nodes. A total of 52 edges in this network had absolute weights greater than zero, that is, roughly $33 \%$ of the theoretical 78 edges had a weight of zero. Notably, approximately all the edges showed positive relationships between nodes; only one edge [average edge weight $(A E W)=-0.091$, see Supplementary Materials] that connected "anxious/depressed problems" (node 8) and "social awareness" (node 1) represented a negative connection. Among all the edges, as depicted in Figure 1, the edge between nodes 5 and 13 ( $A E W=0.369)$ is the strongest, representing the sleep problem assessed by $\mathrm{CBCL}$ and CSHQ, respectively. The second largest edge lay between "emotional problem" (node 7, which belongs to internalizing problem) and "anxious/depressed problems" (AEW $=0.354)$. These two nodes were within the items of $\mathrm{CBCL}$. This was followed by the edges that linked "emotional problem" and "aggressive behavior" (node 13) (AEW = 0.354). Among the edges, the line between "emotional problem" and "social cognition" (node 2) was the thinnest (AEW $=0.004)$.

In addition, apart from the intense relationships with "social awareness" (node 1), "social cognition" (node 2), "social motivation" (node 4), and "restricted interests and repetitive behavior" (node 5, one of the core symptoms in ASD), "social communication problem" (node 3, another core symptom of ASD) had a strong correlation with "withdrawal" (node $10, A E W=0.199$ ). Through node 10 , these nodes could establish indirect contacts with "attention problem" (node 12) and "aggressive behavior" (node 13) (weight of edge between node 10 and node $12=0.304$ ), so did the other associated symptoms of ASD: emotional problems, anxious/depressed problems, sleep problems, and somatic complaints. Meanwhile, "anxious/depressed problems" also had relatively weaker direct relationships with core symptoms. This means that high correlation exists between core and associated symptoms.

There were also broad partial correlations between somatic complaints (node 9) and internalizing problems (nodes 7 and 8), externalizing problems (node 13), and sleep problems on the CBCL scale.

\section{Centrality estimates}


Figure 2 illustrates the centrality estimate index-node strength. Node 3 had the greatest strength estimate among all the nodes (centrality weight $=1.311$ ), which had a much higher node strength than that of all other nodes. That is, node 3 had strong partial correlations with all other nodes. In other words, social communication problems were the most central symptoms. The lowest centrality node was node 9 (somatic complaints, apart from node 6 , which was parallel to node 11 ) (centrality weight $=0.568$ ). As Epskamp's prior work suggested that the centrality estimates indices of closeness and betweenness have relatively poor stability[18], results of these two indices were not exhibited here (see details in Supplementary Materials).

\section{Stability and accuracy of the network}

A bootstrapping procedure was used to assess the stability of the network (Figure 3a). For network edges, the $95 \%$ confidence intervals revealed high stability [Correlation Stability (CS) coefficient $>>0.75$ ]. Among the centrality indices, strength was highly stable (Figure $3 b$ ).

\section{Discussion}

We explored the overall relationship between the symptoms of autism through a large sample of children with autism and an integrative approach-network analysis in this study. Overall, there were broad relationships between the core and associated symptoms included in this study; and almost all of them were positive, with only one negative relationship existed in "anxious/depressed problems" and "awareness." The associations between some of these symptoms were stronger than the other associations. In consistency with the clinical characteristics of ASD, the symptom of "communication" was the most central to the network. The strength centrality and network edges were highly stable, indicating that our results are highly reliable.

\section{Central symptoms in ASD}

The strength centrality index demonstrated excellent reliability; thus, we focus our discussion on symptom centrality on strength.

The social communication problem, the core symptom in ASD, was the most central symptom in our study. Previously, there have been few network analyses on the multidimensional relationship between symptoms of autism. Most of them were compared with other disorders on one or two symptoms, such as depression[19] and obsessive-compulsive disorder[20], or evaluated with associated symptoms of autism[21]. What is consistent with our research results is that, in Anderson's studies about network analysis of autism, "social" and "communication" domains of Autism Diagnostic Observation Schedule (ADOS) ranked top two central items[21]; and the "anxiety" node in both ADOS and Revised Child Anxiety and Depression Scale (RCADS) items were highly peripheral[12, 21], although previous study found a lack of correlation between items scores of CBCL and ADOS[22, 23]. Here, "communication" was the most central node in the network, and "anxious/depressed problems" node in the CBCL items had relatively more peripheral centrality estimates. The lowest centrality node was "somatic complaints", indicating that 
somatic problems were a peripheral symptom in ASD. However, there were quite a number of people with autism who had experienced varied degree of chronic gastrointestinal (GI) problems during different stages of the disorder[24,25], and previous study suggested a interaction between anxiety and GI problems[26]. Additionally, GI problems were associated with externalizing problem behavior and internalizing symptoms, and the relationship varies in different ages of patients[25]. Therefore, further studies are needed to explore the factors of the symptoms' centrality estimates.

\section{Connections among the core and associated symptoms of ASD}

There were broad correlations between core and noncore symptoms in patients with ASD. In this study, a direct relationship was found between anxiety problems and core symptoms, such as restricted interests and repetitive behavior. A recent study indicated an insufficient evidence to support a two-way relationship between anxiety and core symptoms; however, it was also found that anxiety would gradually increase the risk of social communication dysfunction, and the treatment of anxiety symptoms would help improve social and emotional functions[27]. Furthermore, several studies demonstrated that the presence of anxiety and depression symptoms leads to increased severity of ASD symptoms[28, 29]. Similarly, aggressive behaviors and sleep problems had connections with core symptoms and could link via some nodes within the network to the core symptoms. Previous studies suggested that sleep disturbance (such as short sleep duration) was associated with the severity of core symptoms[30] and related symptoms $[8,31]$ in ASD. Additionally, our previous work found that aggressive behaviors were significantly associated with sleep problems and ADHD symptoms, which were treatable[14], and brought new insight into the treatment of aggressive behaviors in ASD.

High comorbidity rates of ADHD were observed across ASD[32]. An increasing amount of evidence suggests that ASD and ADHD may have shared genetic risk patterns[33-36]. Family-based studies show that siblings of patients with ADHD have an increased risk of developing ASD symptoms[34], while studies of twins show that common genetic factors may explain $50 \%$ to $70 \%$ of the covariance between ASD and ADHD symptoms[35, 37]. Here, we found that there were direct or indirect relationships between the characteristic symptoms of ADHD, attention problems, and the core symptoms of ASD-social function and autistic behavior. We can learn that there is also an internal relationship between the clinical phenotypes of ASD and ADHD. Both as neurodevelopmental disorders, ASD may overlap with ADHD in genetic and clinical perspectives that brings new insights into recognizing them.

Relationships within associated symptoms were also found in this study. The edge between sleep problems assessed by $\mathrm{CBCL}$ and $\mathrm{CSHQ}$, in line with expectation, was stronger than all other edges in this network that indicated the high consistency of the two scales and the good sleep status of children with autism. Stronger connections also existed between emotional problems and anxious/depressed problems, emotional problems and aggressive behavior, and withdrawal and attention problems. This means that these symptoms could interact with each other, and a stronger symptom leads to a greater degree of other symptoms. As mentioned earlier, there is a high prevalence of these symptoms in patients with ASD. However, previous studies have not clearly explained the relationship between these symptoms. 
In Duvekot's study, however, results demonstrated that the treatment of anxiety symptoms would help improve emotional function[27]. Furthermore, the only negative connection existed in anxious/depressed problems and social awareness assessed by SRS. Stephen et al. found that ASD patients with higher SRS scores reported greater degree of anxious/depressed problems[38]. Other studies also suggested that social function reflected by SRS scores could predict the severity of anxiety[39, 40]. Mikle et al. found that anxiety symptoms assessed by different scales had significant correlations with the five subscales of SRS, including the social awareness domain[41]. These findings were contrary to our results.

Previous research and clinical evidence show that the treatment of the core symptoms of autism is not effective; whether drug or nondrug treatment, its effect is very small. Other ASD-related symptoms, including attention problems, hyperactivity, anxiety or depression, and sleep disturbance, can also cause clinically significant problems[4]. Many tools can be used to manage these symptoms, such as drug treatment, cognitive-behavioral therapy, and psychosocial treatment[42]. As shown in this study, these ASD-associated symptoms have a complex positive correlation with core symptoms in children with ASD and can interfere with each other, indicating that we can alleviate the core symptoms by treating them and then improve the prognosis of patients, which requires follow-up data for confirmation.

\section{Limitations}

The current study also had some limitations. First, as a cross-sectional study, the network analyses was conducted by using cross-sectional data, so the analysis of some network characteristics is not sufficient. It remains unknown that whether the network structure will change with the progress of the course of the disease or the change of the severity of symptoms. Second, some nodes may actually be measuring overlapping constructs (e.g. "sleep problems" measured by CBCL and CSHQ respectively), which could artificially inflate edge weights and centrality. Third, a group of typically developed children were not enrolled as a controlled sample, and parent questionnairs were used in this study, which may lead to the existence of some confounding factors. In the future, further research can be carried out through a welldesigned follow-up study.

\section{Conclusion}

We conducted a study to explore the holistic relationships of symptoms in a sample with autism using network analysis. Our study found comprehensive relationships between core symptoms and ASD-related symptoms and that they can interfere with each other. These findings indicate that controlling these symptoms may improve core symptoms, such as ADHD-related symptoms. It may help identify new ways to improve symptoms in ASD.

\section{Declarations}

\section{Ethics approval and consent to participate}


This study was approved by the Ethics Committee of the Second Xiangya Hospital. The parents or legal guardians of the subjects who participated in this study signed the consent forms.

\section{Consent for publication}

Not applicable.

\section{Availability of data and materials}

The datasets analysed during the current study are available from the corresponding author on reasonable request.

\section{Competing interests}

The authors declare that they have no competing interests.

\section{Funding}

This study was supported by the National Natural Science Foundation of China (Grant No. 81974217), Major Scientific and Technological Projects for Collaborative Prevention and Control of Birth Defects in Hunan Province (Grant No. 2019SK1015), and the Humanity and Social Science Youth Foundation of the Ministry of Education (Grant No. 17YJC190022).

\section{Authors' contributions}

SY, OJ designed the study. WX, SL, SY, OJ performed the data analyses and wrote the draft of this manuscript. WX, SL, WY, LJ, CX and LR conducted the clinical assessments. WR, XK and ZJ managed the data collection. All authors contributed to the editing of the manuscript and agreed with the final text.

\section{Acknowledgments}

We are very grateful to all the researchers involved in data collection as well as to the children and parents who volunteered to participate in this study.

\section{Abbreviations}

ASD: Autism Spectrum Disorder; PDD: pervasive developmental disorders; ADHD: attention-deficit hyperactivity disorder; PDD-NOS: pervasive developmental disorder not otherwise specified; DSM-IV-TR: Diagnostic and Statistical Manual of Mental Disorders, Fourth Edition, Text Revised; SRS: Social Responsiveness Scale; CSHQ: Children's Sleep Habit Questionnaire; CBCL: Child Behavior Checklist; GGM: graphical Gaussian model; AEW: average edge weight; ADOS: Autism Diagnostic Observation Schedule; RCADS: Revised Child Anxiety and Depression Scale; GI: gastrointestinal.

\section{References}


1. American Psychiatric Association. Diagnostic and statistical manual of mental disorders. 5th ed. Arlington: American Psychiatric Association; 2013.

2. Hodge D, Carollo TM, Lewin M, Hoffman CD, Sweeney DP. Sleep patterns in children with and without autism spectrum disorders: developmental comparisons. Res Dev Disabil. 2014;35(7):1631-8.

3. Maskey M, Warnell F, Parr JR, Le Couteur A, McConachie H. Emotional and behavioural problems in children with autism spectrum disorder. J Autism Dev Disord. 2013;43(4):851-9.

4. Lecavalier L. Behavioral and emotional problems in young people with pervasive developmental disorders: relative prevalence, effects of subject characteristics, and empirical classification. J Autism Dev Disord. 2006;36(8):1101-14.

5. Lord C, Elsabbagh M, Baird G, Veenstra-Vanderweele J. Autism spectrum disorder. Lancet. 2018;392(10146):508-20.

6. Richards C, Oliver C, Nelson L, Moss J. Self-injurious behaviour in individuals with autism spectrum disorder and intellectual disability. J Intellect Disabil Res. 2012;56(5):476-89.

7. Hill AP, Zuckerman KE, Hagen AD, Kriz DJ, Duvall SW, van Santen J, et al. Aggressive Behavior Problems in Children with Autism Spectrum Disorders: Prevalence and Correlates in a Large Clinical Sample. Res Autism Spectr Disord. 2014;8(9):1121-33.

8. Cohen S, Conduit R, Lockley SW, Rajaratnam SM, Cornish KM. The relationship between sleep and behavior in autism spectrum disorder (ASD): a review. J Neurodev Disord. 2014;6(1):44.

9. Bos MGN, Diamantopoulou S, Stockmann L, Begeer S, Rieffe C. Emotion Control Predicts Internalizing and Externalizing Behavior Problems in Boys With and Without an Autism Spectrum Disorder. J Autism Dev Disord. 2018;48(8):2727-39.

10. Gray K, Keating C, Taffe J, Brereton A, Einfeld S, Tonge B. Trajectory of behavior and emotional problems in autism. Am J Intellect Dev Disabil. 2012;117(2):121-33.

11. Cramer AO, Waldorp LJ, van der Maas HL, Borsboom D. Comorbidity: a network perspective. Behav Brain Sci. 2010;33(2-3):137 - 50; discussion 50-93..

12. Anderson GM, Montazeri F, de Bildt A. Network approach to autistic traits: group and subgroup analyses of ADOS item scores. J Autism Dev Disord. 2015;45(10):3115-32.

13. Beard C, Millner AJ, Forgeard MJ, Fried El, Hsu KJ, Treadway MT, et al. Network analysis of depression and anxiety symptom relationships in a psychiatric sample. Psychol Med. 2016;46(16):3359-69.

14. Chen C, Shen YD, Xun GL, Cai WX, Shi LJ, Xiao L, et al. Aggressive behaviors and treatable risk factors of preschool children with autism spectrum disorder. Autism Res. 2017;10(6):1155-62.

15. John N. Constantino SAD, Richard D, Todd MK, Schindler MM, Gross SL, Brophy LM, Metzger CS, Shoushtari. Reagan Splinter, and Wendy Reich. Validation of a Brief Quantitative Measure of Autistic Traits: Comparison of the Social Responsiveness Scale with the Autism Diagnostic InterviewRevised. J Autism Dev Disord. 2003;33(4):427-33. 
16. Owens JA, Spirito A, McGuinn M. The Children's Sleep Habits Questionnaire (CSHQ): psychometric properties of a survey instrument for school-aged children. Sleep. 2000;23(8):1043-51.

17. Achenbach TMaLAR. Manual for the ASEBA Preschool Forms \& Profiles: An integrated system of multi-informant assessment; Child Behavior Checklist for Ages 1 1/2-5; Language Development Survey; Caregiver-teacher Report Form. Burlington: University of Vermont, Research Center for Children, Youth, \& Families; 2000.

18. Epskamp S, Borsboom D, Fried El. Estimating psychological networks and their accuracy: A tutorial paper. Behav Res Methods. 2018;50(1):195-212.

19. Montazeri F, de Bildt A, Dekker V, Anderson GM. Network Analysis of Behaviors in the Depression and Autism Realms: Inter-Relationships and Clinical Implications. J Autism Dev Disord. 2019.

20. Ruzzano L, Borsboom D, Geurts HM. Repetitive behaviors in autism and obsessive-compulsive disorder: new perspectives from a network analysis. J Autism Dev Disord. 2015;45(1):192-202.

21. Montazeri F, de Bildt A, Dekker V, Anderson GM. Network Analysis of Anxiety in the Autism Realm. J Autism Dev Disord. 2019;49(6):2219-30.

22. Hartley SL, Sikora DM, McCoy R. Prevalence and risk factors of maladaptive behaviour in young children with Autistic Disorder. J Intellect Disabil Res. 2008;52(10):819-29.

23. Guerrera S, Menghini D, Napoli E, Di Vara S, Valeri G, Vicari S. Assessment of Psychopathological Comorbidities in Children and Adolescents With Autism Spectrum Disorder Using the Child Behavior Checklist. Front Psychiatry. 2019;10:535.

24. Buie T, Campbell DB, Fuchs GJ 3rd, Furuta GT, Levy J, Vandewater J, et al. Evaluation, diagnosis, and treatment of gastrointestinal disorders in individuals with ASDs: a consensus report. Pediatrics. 2010;125(Suppl 1):1-18.

25. Ferguson BJ, Dovgan K, Takahashi N, Beversdorf DQ. The Relationship Among Gastrointestinal Symptoms, Problem Behaviors, and Internalizing Symptoms in Children and Adolescents With Autism Spectrum Disorder. Front Psychiatry. 2019;10:194.

26. Mazurek MO, Vasa RA, Kalb LG, Kanne SM, Rosenberg D, Keefer A, et al. Anxiety, sensory overresponsivity, and gastrointestinal problems in children with autism spectrum disorders. J Abnorm Child Psychol. 2013;41(1):165-76.

27. Duvekot J, van der Ende J, Verhulst FC, Greaves-Lord K. Examining bidirectional effects between the autism spectrum disorder (ASD) core symptom domains and anxiety in children with ASD. J Child Psychol Psychiatry. 2018;59(3):277-84.

28. Kelly AB, Garnett MS, Attwood T, Peterson C. Autism spectrum symptomatology in children: the impact of family and peer relationships. J Abnorm Child Psychol. 2008;36(7):1069-81.

29. Sterling L, Dawson G, Estes A, Greenson J. Characteristics associated with presence of depressive symptoms in adults with autism spectrum disorder. J Autism Dev Disord. 2008;38(6):1011-8.

30. Veatch OJ, Sutcliffe JS, Warren ZE, Keenan BT, Potter MH, Malow BA. Shorter sleep duration is associated with social impairment and comorbidities in ASD. Autism Res. 2017;10(7):1221-38. 
31. Gabriels RL, Cuccaro ML, Hill DE, Ivers BJ, Goldson E. Repetitive behaviors in autism: relationships with associated clinical features. Res Dev Disabil. 2005;26(2):169-81.

32. Leyfer OT, Folstein SE, Bacalman S, Davis NO, Dinh E, Morgan J, et al. Comorbid psychiatric disorders in children with autism: interview development and rates of disorders. J Autism Dev Disord. 2006;36(7):849-61.

33. Ghirardi L, Pettersson E, Taylor MJ, Freitag CM, Franke B, Asherson P, et al. Genetic and environmental contribution to the overlap between ADHD and ASD trait dimensions in young adults: a twin study. Psychol Med. 2019;49(10):1713-21.

34. Mulligan A, Anney RJ, O'Regan M, Chen W, Butler L, Fitzgerald M, et al. Autism symptoms in Attention-Deficit/Hyperactivity Disorder: a familial trait which correlates with conduct, oppositional defiant, language and motor disorders. J Autism Dev Disord. 2009;39(2):197-209.

35. Ronald A, Simonoff E, Kuntsi J, Asherson P, Plomin R. Evidence for overlapping genetic influences on autistic and ADHD behaviours in a community twin sample. J Child Psychol Psychiatry. 2008;49(5):535-42.

36. Xia L, Ou J, Li K, Guo H, Hu Z, Bai T, et al. Genome-wide association analysis of autism identified multiple loci that have been reported as strong signals for neuropsychiatric disorders. Autism Res. 2020;13(3):382-96.

37. Reiersen AM, Constantino JN, Grimmer M, Martin NG, Todd RD. Evidence for shared genetic influences on self-reported ADHD and autistic symptoms in young adult Australian twins. Twin Res Hum Genet. 2008;11(6):579-85.

38. Kanne SM, Christ SE, Reiersen AM. Psychiatric symptoms and psychosocial difficulties in young adults with autistic traits. J Autism Dev Disord. 2009;39(6):827-33.

39. White SW, Kreiser NL, Pugliese C, Scarpa A. Social anxiety mediates the effect of autism spectrum disorder characteristics on hostility in young adults. Autism. 2012;16(5):453-64.

40. Duvekot J, van der Ende J, Constantino JN, Verhulst FC, Greaves-Lord K. Symptoms of autism spectrum disorder and anxiety: shared familial transmission and cross-assortative mating. J Child Psychol Psychiatry. 2016;57(6):759-69.

41. South M, Carr AW, Stephenson KG, Maisel ME, Cox JC. Symptom overlap on the srs-2 adult self-report between adults with asd and adults with high anxiety. Autism Res. 2017;10(7):1215-20.

42. Aman MG, Lam KS, Van Bourgondien ME. Medication patterns in patients with autism: temporal, regional, and demographic influences. J Child Adolesc Psychopharmacol. 2005;15(1):116-26.

\section{Tables}

Table 1. Demographic and Clinical Characteristics of Participates $(n=474)$ 


\begin{tabular}{|ll|}
\hline Variable & ASD (n=474) \\
\hline M (SD) \\
\hline Age (year) & $402 / 72$ \\
\hline SRS & $3.73(1.01)$ \\
\hline SRS Total scores & \\
\hline Awareness & $93.26(22.23)$ \\
\hline Cognition & $13.46(2.91)$ \\
\hline Communication & $18.26(4.70)$ \\
\hline Motivation & $32.92(8.44)$ \\
\hline Restricted interests and repetitive behavior & $15.50(4.46)$ \\
\hline CSHQ Total score & $13.13(6.13)$ \\
\hline CBCL (1.5-5) & $46.48(6.25)$ \\
\hline Total problems & \\
\hline Internalizing problems & $56.70(10.05)$ \\
\hline Emotional problem & $59.68(9.29)$ \\
\hline Anxious/depressed problems & $57.00(7.96)$ \\
\hline Somatic complaints & $54.90(6.09)$ \\
\hline Withdrawal & $54.57(6.46)$ \\
\hline Sleep problem & $52.25(10.88)$ \\
\hline Externalizing problems & $54.04(5.96)$ \\
\hline Attention problem & $51.95(8.67)$ \\
\hline Aggressive behavior & $59.88(7.47)$ \\
\hline
\end{tabular}

Abbreviations: SRS, Social Responsiveness Scale; CBCL/1.5-5, Child Behavior Checklist version for preschool children; CSHQ, Children's Sleep Habits Questionnaire

\section{Figures}




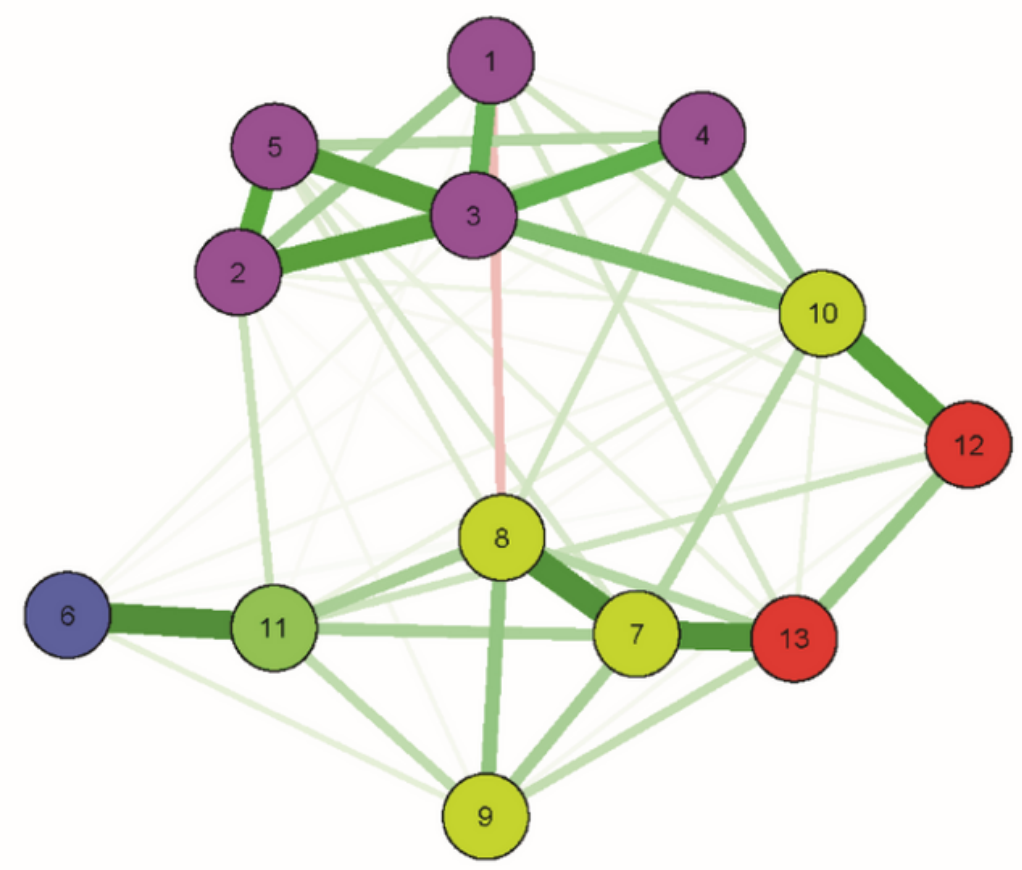

SRS

- 1: Awarenness

- 2: Cognition

- 3: Communication

- 4: Motivation

- 5: Restricted Interests and Repetitive Behavior

CSHQ

- 6: CSHQ tota

CBCL Internalizing

- 7: Emotional Problem

8: Anxious/Depressed

9: Somatic Complaints

- 10: Withdrawn

CBCL Sleep Problem

- 11: Sleep Problem

CBCL Externalizing

12: Attention Problem

- 13: Aggressive Behavior

\section{Figure 1}

The network structure of ASD sample.

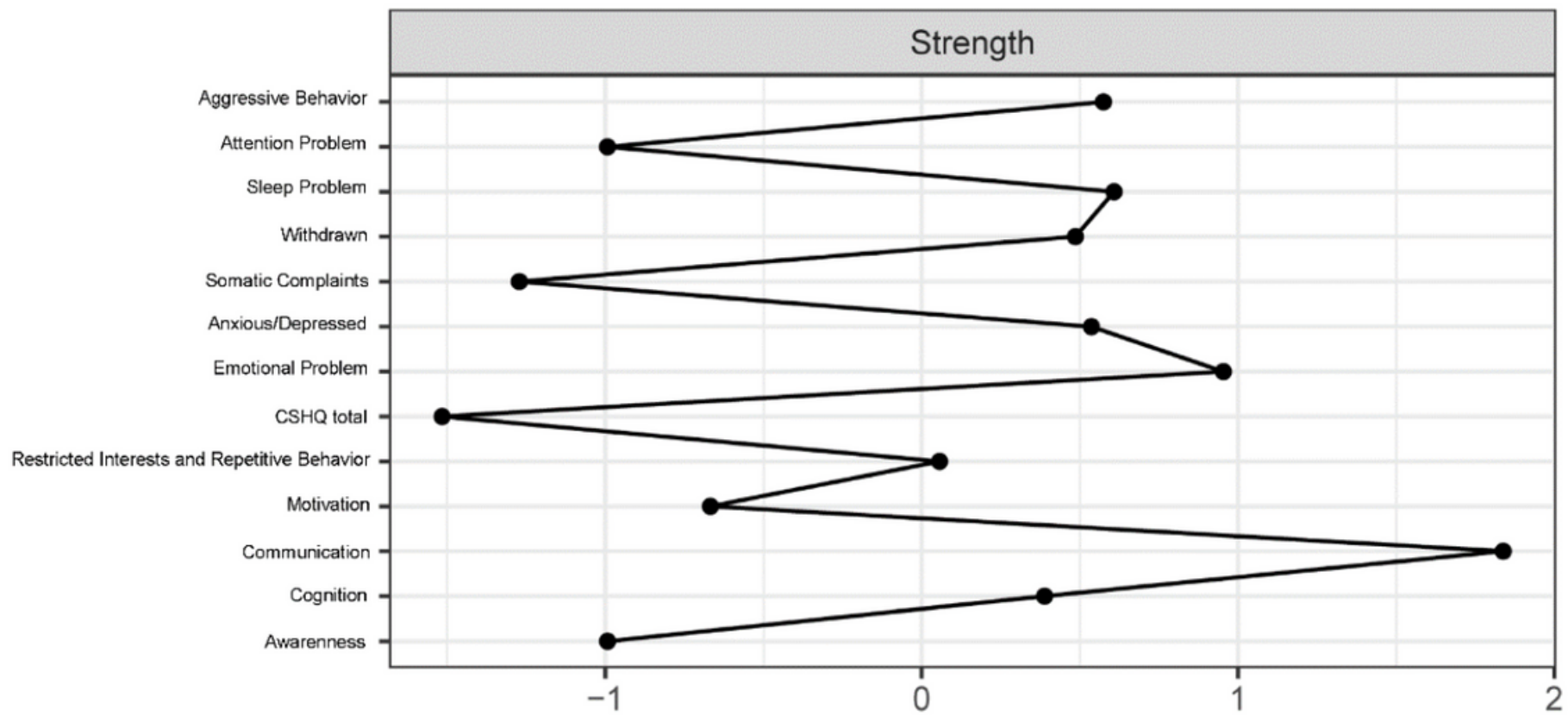

Figure 2 
Centrality estimate index-node strength of the network.

\section{- Bootstrap mean $\bullet$ Sample}
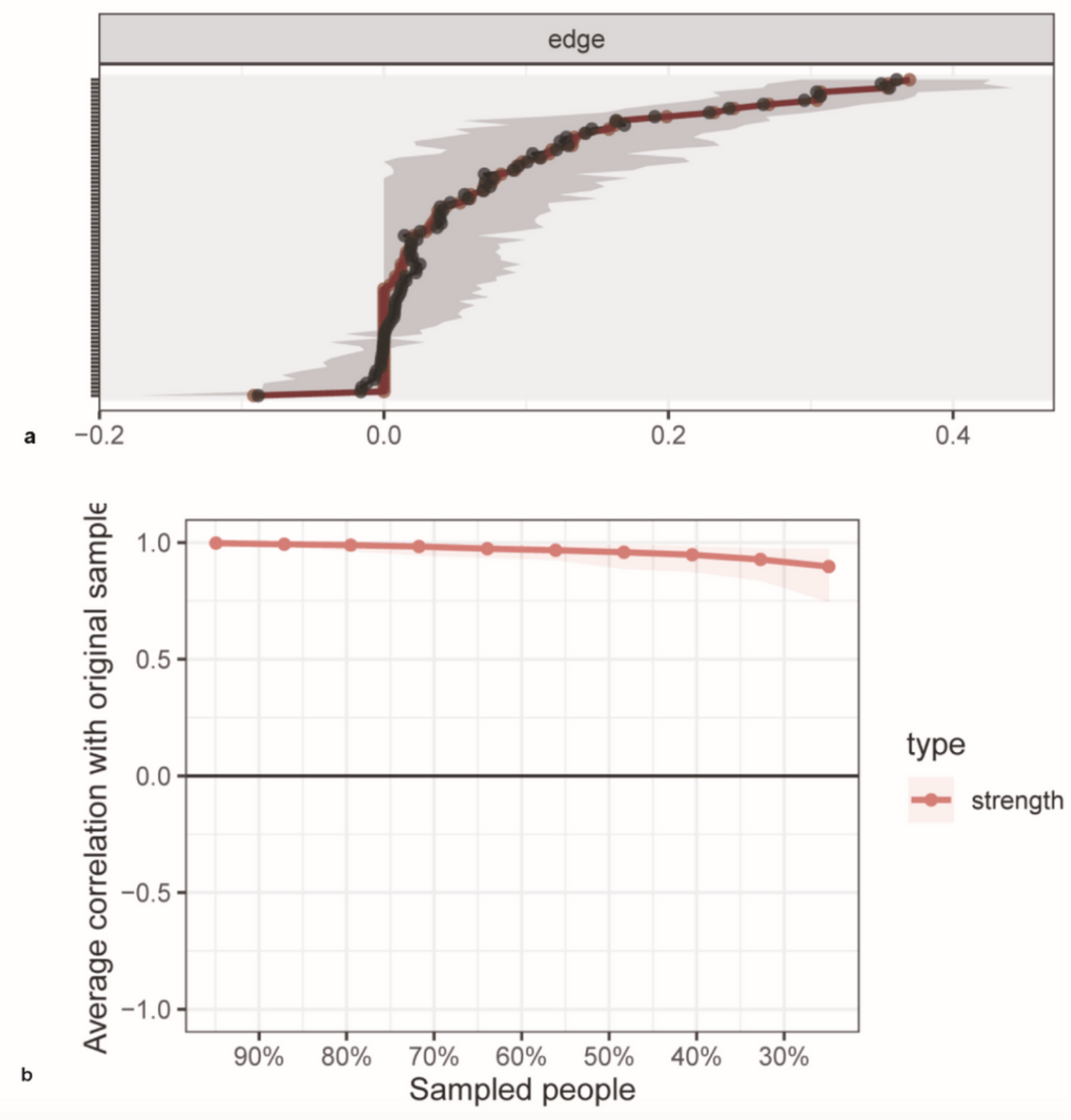

\section{Figure 3}

a) The bootstrap confidence intervals of estimated edge-weights for the network of core symptoms and noncore symptoms in ASD. Each horizontal line represents one edge of the network, ordered from the edge with the highest edge-weight to the edge with the lowest edge-weight. Y-axis labels have been removed. b) Average correlations between centrality index-strength of networks with sampled persons 
dropped and the original sample. Lines indicate the means, and areas indicate the range from the 2.5 th quantile to the 97.5 th quantile.

\section{Supplementary Files}

This is a list of supplementary files associated with this preprint. Click to download.

- SupplementaryMaterials.docx 